\title{
A Review on the Reproduction and Development in Fish
}

\author{
Chattopadhyay $\mathrm{NR}^{1 *}$ and Chattoraj $\mathrm{S}^{2}$ \\ ${ }^{1}$ Department of Zoology, Rajiv Gandhi University, India \\ ${ }^{2}$ Baharampur Girls College, India
}

Received: October 27,2017; Published: November 14, 2017

*Corresponding author: Chattopadhyay NR, Department of Zoology, Rajiv Gandhi University, Rono Hills, Doimukh, Tanager 791112, Arunachal Pradesh, India; Email: nrchatterjee40@gmail.com

\section{Review}

In order that survival of young be more than optimum, the timing of spawning by the mature adult fish is so adjusted that must be closely linked to the availability of maximum congenial environment added by increased food availability of food for the newly hatched young ones. The availability of food for the larvae and juveniles of most temperate and cold water fish species varies on a seasonal basis and following this fish are usually found to have a discrete and selective spawning season so perfectly timed that hatched out larvae will get readily available exogenous food from nature for their further development. Thus, in many temperate and cold water species, spawning is an annual event. Spawning culminates a series of preparatory events during which the gametes develop and the gonads increase in size. The annual cycle of fishesgenerally categorized into three major phases:

A. Post Spawning phase: Period when the gonads are thin and slender and appear to be in a resting phase.

B. Pre Spawning phase: Period in which the gonads begin production of gametes (gameto genesis) and incorporation of yolk into the oocytes (vitello genesis) which is accompanied by a process known as gonadal recrudescence i.e. gradual increase in gonad size.

C. Spawning phase: Period involving final maturation and ripening of the gametes; this phase culminates in spawning act, with the release of egg and milt which ensures fertilization.

Thus, gonadal development is considered to encompasses a series of interrelated phases, each requiring precise coordination and control if viable gametes are to be produced at the optimum time of the year for the subsequent survival of the young fish. The production of gametes involves the manufacture and incorporation of yolk into the developing oocytes (vitello genesis). During the early part of gonadal development, the oogonia, through the process of oogenesis produce mature eggs. Primarily, under the influence of GTHI, primary oogonium forms several primary follicles which ultimately develop into mature graffian follicle. It is within this graffwian follicle eggs mature and get associated with vitallogenin protein from liver known as yolk granule, acts as a readymade food for the growing embryo during development of larvae. Supported by this endogenous food gamete grow in size during post vitallogenic phase of development alin particular. In the salmonids, for example, the oocytes increase in diameter from about $50 \mu \mathrm{m}$ to $500-1000 \mu \mathrm{m}$ prior to the start of vitellogenesis. The incorporation of yolk that occurs during the vitellogenic phase leads to the diameter of the oocyte increasing to around $5000 \mu \mathrm{m}(5 \mathrm{~mm})$. Thus, there is an enormous increase in oocyte volume during post vitellogenic phase.

This observation supports the view that in majority of fishesthe gametic development is dependent on endogenous hormone and the nutrient protein from liver and its subsequent incorporation into egg cytoplasm. The yolk is derived from vitellogenin, a lipophosphorotein- calcium complex with a molecular weight of approximately 440,000. Vitellogenin is synthesized in the liver and is then released into the blood system. The vitellogenin is then transported to the ovary through hepatic portal blood vessel from which it is sequestered by the oocyte. Following uptake into the oocyte the vitellogenin splits into two major components each having a molecular weight of 35000 , which form the yolk stores. The processes of gametic development and gonadal growth usually occur gradually and the prespawning phase of the cycle often continues for a considerable period of time, perhaps several months, before the actual spawning occurs. These indicate that the fish can 'predict' the arrival of spawning time several months in advance.

\section{Environmental Influences on the Reproductive Cycle}

In general, growth and development of gamete depends on different environmental cues in nature. The primary environmental cues are often linked to the annual cycles of day length and temperature variationsm Chattopadhyay et al. [1]. For example, in case of Cyprinus carpio and many other cyprinids, gonadal maturation begins in late winter after the vernal equinox $\left(21^{\text {st }}\right.$ March) when day length is in increasing manner and as a result water temperature is also in increasing trend. But in summer when temperature is high, cessation of spawning activity may occur due to inhibitory effects of high water temperature. Thus, it is thought that both photoperiod and temperature are major environmental cues responsible for the mediation of the reproductive cycle in 
several species of fish that inhabit temperate latitudes Devlin $\mathrm{RH}$, Nagahama Y [2]. In temperate area salmon generally breed at temperature range of 8-10 oC i.e. during late autumn and early winter, but during autumn and winter when temperature of river water fal1.sto 1-2 oC, inhibition of oocyte growth and development may occur. In contrast to this fish are maintained under experimental condition of constant photoperiod (e.g. 12L: $12 \mathrm{D}-12 \mathrm{~h}$ light and $12 \mathrm{~h}$ dark each day) and temperature) the fish may still spawn at approximately yearly intervals. This means there is a strong autonomous component to the reproductive cycle, and under conditions this endogenous rhythm will have a periodicity of about 1 year.

In constant environment condition, fishes may spawn at intervals that are approximately but are significantly different from 1 year. Thus the periodicity of the rhythm is only approximately 1 year, i.e. it is circannual. As a result, many fish species rely on the seasonally changing cycle of day length to match their annual cycles of reproduction and these cyclic events will be mediated through a number of endocrine changes. Consequently, it is ultimately the neuro-endocrine system which directly controls the different phases of general development and the maturation of gametes. The general environmental cues that triggers maturation, gametice growth and development appears to be primarily environmental in nature followed subsequent endogenous rhythm. These environmental cues are often linked to the annual cycles of day length and temperature variations. For example, for in summer-spawning fish such as carp, Cyprinus carpio, and many other cyprinids, gonadal maturation begins in late winter or early spring. The start of the maturation process is thought to be triggered by a combination of increasing day length and the rise in water temperature with the commencement of spring. The extended spawning season, with a startup season in March-April continues up to the end of August through scientific management practice. Some authors are of the idea opinion that in certain fishes' cessation of spawning activity may, in part, be due to inhibitory effects of high summer water temperatures on gonadal differentiation and maturation. For tropical fishes' gonadal development and spawning stops with the fall of temperature that happen with the approach of winter. This provides a direct evidence that temperature along with photoperiod is the main controlling factor towards maturation and spawning in fish. S.

Again, some species such as the Tobinumeri dragonet and Repomucenusbeniteguri, resume spawning activities as water temperature falls during the course of autumn. These fish exhibit two distinct spawning seasons (May-July and SeptemberNovember) during the course of the year. Spawning commences in spring when the water temperature reaches $18^{\circ} \mathrm{C}$, but the spring spawning season comes to an end once temperature exceeds $28^{\circ}$ C. Spawning that resumes in September when water temperature falls below $27^{\circ} \mathrm{C}$, and the autumn spawning season continues as long as temperature stays above $15^{\circ} \mathrm{C}$. Thus, gonadal development and maturation appear to be inhibited by temperatures lower than about $15{ }^{\circ} \mathrm{C}$ and higher than $27-28{ }^{\circ} \mathrm{C}$. This suggests that water temperature is probably the major environmental cue for both inhibition and termination of the spawning seasons in the Tobinumeri dragonet. In the tropics changes in photoperiod and water temperature occurs after the vernal equinox that falls on $21^{\text {st }}$ March every year. Gonadal recrudescence starts after this period for most of the fishes. Though these two environmental factors are unlikely to act as major cues for influencing the reproductive cycle in some fishes like tilapia and Cyprinus carpio. In some tropical species there does not appear to be any distinct spawning season and some members of the population can be found to be engaged in reproductive activities at almost any time of the year. In other species, spawning activities do seem to vary on a seasonal basis, with factors such as variations in rainfall and humidity possibly acting as the environmental cues triggering changes in gonadal development and maturation.

\section{Endocrine Regulation of Fish Gonad Development}

The development and maturation of fish gonads are controlled by genetic and environmental factors, and regulated by a molecular network of signals. As in other vertebrates, the HPG axis of the neuroendocrine system plays a central regulatory role on the reproductive development of fishes. Gonadotropin-releasing hormone (GnRH), lying upstream of the HPG axis, is a key regulatory factor. Once hypo- thalamus-secreted GnRH arrives at the pituitary gland, the cAMP signal pathway in gonadotrophs will be activated, transcription, synthesis and secreting of FSH and LH are all upregulated. Via blood circulation, FSH and LH enter the gonads to regulate gonad development and to promote sexual maturation by stimulating production of sex steroids. On the other hand, sex steroids can inhibit synthesis of GnRH in the hypothalamus through a negative feedback pathway. In recent years, the discoveries of kisspeptin, which can positively regulate GnRH, and GnIH, which can negatively regulate $\mathrm{GnRH}$, have been praised as two remarkable breakthroughs in vertebrate neuro endocrinology Zohar Y [3]. They have become attractive topics in fish reproduction and physiological research.

Kisspeptin is a neuropeptide encoded by the kiss gene and act in conjugation with GPR54 as the receptor. The first research about kiss function was carried out in hypogonadism De Roux N [4]. The patients had delayed pubertal maturation and reproductive function due to a mutation in the gene encoding GPR54. Subsequent studies in mice, rats and monkeys further confirmed the functions of kiss peptin and GPR54 in reproductive development. Currently kiss peptin is thought to play a significant role on sexual differentiation of the brain, the initiation of puberty, secretion and release of GnRH, transmission of feedback signals of sex steroids and control of reproductive function by a photoperiodic factor Tena-Sempere M [5]. Currently, functional research of Kiss peptin and GPR54 in fish reproduction is developing rapidly. Parhar [6] found mRNA of GPR54 in GnRH neurons of tilapia, which suggested a direct effect of kisspeptin on GnRH neurons. Kitahashi [7] cloned and expressed the Kiss2 gene of zebrafish and medaka. Via intraperitoneal administration and real-time PCR, they observed a more important role of kiss2 than kiss1 in regulating zebra fish sex steroid synthesis. Li S [8] observed elevated levels of LH in goldfish serum after intraperitoneal administration of mature kisspeptin 
peptides. However, Pasquier [9] confirmed an inhibitory effect of kisspeptin on LH levels of the eel, Anguilla anguilla. It is worth noting that two or three types of GnRH usually coexist in one fish species, and that two types of kisspeptin and GPR54, respectively, are also found. Thus, the relationship between the kisspeptin/ GPR54 signal system and GnRH, as well as regulatory mechanisms on reproduction, might differ between fishes and mammals.

GnIH (gonadotropin-inhibitory hormone) was first found in birds by Japanese scientists in 2000. Subsequent studies showed that $\mathrm{GnIH}$ might regulate reproduction of birds and mammals on every level of the HPG axis. It can inhibit synthesis and release of LH and FSH, induce apoptosis of testis cells, and might have a regulatory effect on the production of sex steroids as well as on the differentiation and maturation of germ cells by the way of autocrine or paracrine mechanisms Tsutsui K [10]. The functional study of GnIH in fishes has just started, and there are still many inconsistent findings. For instance, Zhang [11] detected lower levels of LH in the serum of mature female goldfish after intraperitoneal administration of zebra fish GnIH peptides, which demonstrated that GnIH could negatively regulate release of LH in goldfish. On the contrary, the results from intraperitoneal administration of GnIH to goldfish at developmental stages from early to late gonad recrudescence showed that GnIH increased pituitary LH- $\beta$ and FSH- $\beta$ mRNA levels as well as reduced serum LH and pituitary GnIH-R mRNA levels Moussavi M et al. [12] It has been suggested that GnIH has a seasonal effect on LH and FSH. This could change our understanding of the regulation of fish re- productive axis. It would also help us to study the function of GnIH in fish reproduction and explore new factors negatively regulating $\mathrm{GnIH}$.

There are also types of molecules that can indirectly regulate fish reproductive development by affecting the HPGaxis. For example, in many teleosts the release of GtH can be inhibited by dopamine (DA) Trudeau VL [12], or be accelerated by $\gamma$-aminobutyric acid (GABA) Popesku JT [13], and secretion of GtH can be stimulated by neuropeptide Y (NPY) Zhang Y [11]. In our group, suppression subtractive hybridization (SSH) and cDNA microarray were applied to sterile females of the common carp with abnormal gonads and to wild type common carp Xu J [14-16]. We found more than 200 genes expressed differently at the hypothalamus, pituitary or gonad level, which included 87 unknown genes, whereas the other genes were associated with growth, organogenesis, energy metabolization, immune response, signal transduction and cell apoptosis. The results showed a very complex molecular network regulating fish reproductive development.

\section{Fertilization and Post-Embryonic Development}

Following fertilization, zygote undergoes meroblastic cleavage to form a ball of cells called morula, which subsequently transformed into blastula (Figures 1 \& 2). The blastula, consists of a central dorsal blastodisc above the yolk sac, through continuous division forms three layer above the yolk - indicate the initiation gastrulation or cell movement. The dorsal layers, which contribute to the formation of embryo proper, by way of differentiation form a dorsal epiblast and ventral hypoblast. Below the hypoblast, a layer composed of loosely packed cell mass remains covering the yolk, called periblast. Gastrulation in bony fishes is accomplished by two processes; invagination and epiboly.At the beginning of gastrulation, the presumptive endodermal and mesodermal cells at the posterior and posterolateral end of the blastoderm turn inwards and migrate forwards under the blastoderm, so as to form the hypoblast. However, there is no in pushing of the epithelial layer and no true archenteron is formed. The presumptive endodermal cells lying along the posterior margin of the blastoderm, migrate inward along the surface of the yolk.

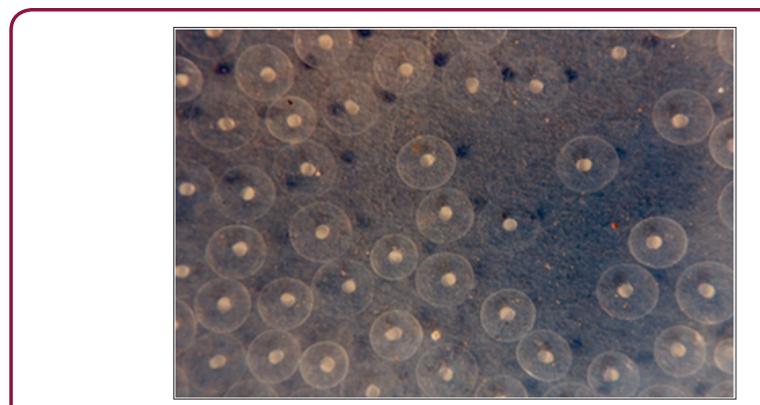

Figure 1: Water hardened eggs with developing eggs within.

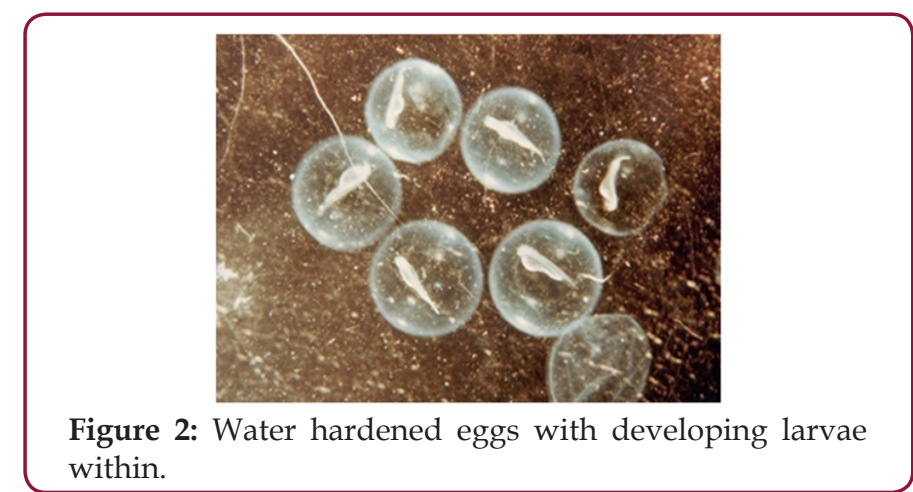

As these cells move inwards, they tend to be concentrated towards the middle of the blastoderm. The presumptive mesoderm cells also invaginate in a similar way and roll over the edge of the blastoderm. Although no true archenteron is formed, the edge of the blastoderm may be compared with the dorsal lip of the blastopore of amphibians. The mesodermal cells, after invagination, also converge towards the middle line, where the axial organs of the embryo are formed. Next, the precordial plates as well as the notochord cells also move inwards by rolling over along the posterior edge of the blastoderm, which corresponds with the dorsal lip of the blastopore. Notochord, prechordal plate and mesoderm are continuous with each other end, form the chord mesoderm. Initially, this involuted cell mass is called the hypoblast. The presumptive neural plate is now started stretching towards the posterior edge of the blastoderm and comes to occupy the areas vacated by the cells that have migrated to the inside. The presumptive neural cells also tend to be concentrated towards the midline and form a strip of tissue lying above the notochord and extend forward to from the posterior edge of the blastoderm. A peculiar feature in the development of fishes is that the formation of the primary organ rudiments begins in the anterior part of the embryo, long before the invagination is completed in the posterior 
part. The anterior part of the neural plate, corresponding to the forebrain appears first, and is followed by the mid brain region. The notochord gradually separated from the mesoderm, which becomes thickened due to concentration towards the midline, and is segmented into somite's lying on either side of the notochord. The rest of the mesoderm remains un segmented and is called the lateral plate mesoderm. The endoderm also becomes separated from the mesoderm and endodermal plate in the middle line. This later forms the alimentary canals.

The process of neurolation in fishes is not like that of the amphibians. The neural plate does not roll into a tube but narrows gradually and sinks deeper into the underlying tissue. It then separates from epidermis, which grows over it and becomes continuous to form the dorsal surface of the embryo. The neural cavity is not formed by rolling over of the neural plate, but it appears later in the brain and the spinal cord by separation of cells in their middle region. During the process of involution, the cells of the blastoderm continue to grow over the yolk through the process of epiboly. During this, the presumptive ectoderm cells grow over and cover the yolk mass from outside, forming a layer of cells called epiblast. At the same time the periblast also grows and forms an inner covering of the yolk. The periblast and epiblast enclose the yolk in a yolk sac. Gastrulation is completed with the formation of yolk sac. At this stagethe the embryo proper can easily be distinguished from yolk sac.

\section{Hatching and Post-Embryonic Development}

After the completion of gastrulation, various organs of the body are formed resulting in a small embryo with more or less cylindrical and bilaterally symmetrical body. The body is raised up from the surface of the yolk, so that the embryo proper becomes distinct from the yolk sac. In this condition, the head of the embryo projects anteriorly from the yolk sac, the trunks lie over the yolk and the tail projects behind. The broad connection between the body and the yolk sac becomes constricted so as to form a stalk. Blood vessels develop in the wall of the yolk sac. Yolk is digested by the periblast and supplied to the body through blood vessels. The yolk sac gradually reduced in size and the embryo grows indicating the supply of nutrients to the growing embryo. Finally, on hatching a free swimming larva comes out. During this period of development, various organs of the body are formed from the ectoderm, mesoderm and endoderm. The ectoderm gives rise to the epidermis and its derivatives like the enamel of the teeth, olfactory epithelium, lens of the eye and the inner ear.

The neural plate which is also ectodermal in origin and gives rise to the brain, spinal cord, retina etc.The mesoderm divides into three parts- dorsal epimere, middle mesomere and ventral hypomere. The dorsal epimere becomes divided into smites at a very early stage. Each somite is subdivided into three parts- sclerotone, myotome and dermatome. The sclerotome collects round the notochord to form the vertebral column. The myotome gives rise to the muscles of the trunk, appendicular skeleton, appendages and their muscles. The dermatome gives rise to the connective tissue and muscles of the dermis and its derivatives i.e., scales. The middle part of the mesoderm (mesomere) gives rise to the kidneys, gonads and their ducts. The hypomere splits into somatic and splanchnic layers of the mesoderm and enclose the coelomic cavity. The splanchnic layers form the mesenchyme which forms the involuntary muscles and connective tissue of the gut. Heart and blood vessels also develop from the splanchnic mesoderm. Skeleton and musculature of the head, outer layers of the eye and the dentine of the teeth develop from the mesenchyme of the head region.

\section{Larval Development}

Young stages of fish, from hatchling stage to till they attain maturity are known as larvae or fry. The larval development period varies considerably in different fishes. A fish fry with a yolk sac (Figure 3) is known as the 'sac fry' but when the sac disappeared is known as 'advanced fry. In some species the advanced fry closely resembles adult and develop into adult without any larval stage. This is called direct development and is seen in salmon, trout and many catfishes. On the other hand, an indirect development through larval stages takes place in several families of fishes like the Clupeidae, Cyprinidae, Lophiidae, Anguillidae, Scorpaenidae etc. In these fishes the larvae (fry) undergoes metamorphosis during which the larval characters are lost and the adult features appear. Through this development the larvae attain a miniature adult like appearance called 'fingerling'. This enters into an active feeding stage which results in maturation of gonads to give rise to the adult. The females are usually larger in size than the males but the latter attain early sexual maturity.

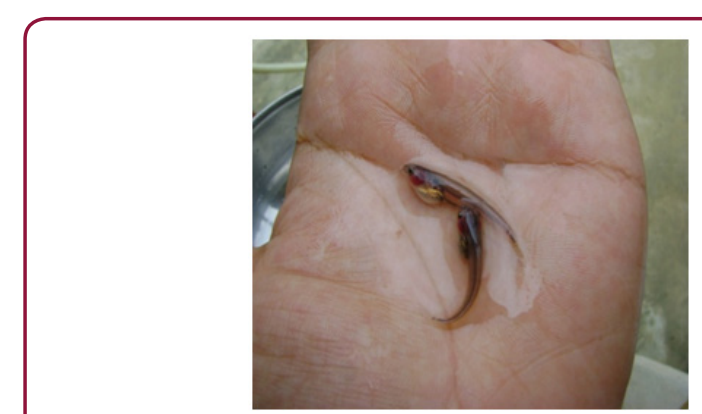

Figure 3: Advanced sac fry.

\section{References}

1. Chattopadhyay (2016) Photo-Thermal and Hormonal Regulation On Maturation Andspawning In Fish. International Journal of Recent Scientific Research 7(2): 8714-8717.

2. Devlin RH, Nagahama Y (2002) Sex determination and sex differentiation in fish: An overview of genetic, physiological, and environmental influences. Aquaculture 208: 191-364.

3. Zohar Y, Muñoz-Cueto JA, Elizur A, Kah O (2010) Neuroendocrinology of reproduction in teleost fish. Gen Comp Endocrinol 165(3): 438-455.

4. CHEN Ji, HU Wei, ZHU Zuo Yan (2012) Progress in studies of fish reproductive development regulation. Chin Sci Bull 58(1): 1-16.

5. Tena-Sempere M, Felip A, Gómez A, Zanuy S, Carrillo M, et al. (2012) Comparative insights of the kisspeptin/kisspeptin receptor system: Lessons from non-mam- malian vertebrates. Gen Comp Endocrinol 175(2): 234-243.

6. Parhar IS, Ogawa S, Sakuma Y (2004) Laser-captured single digoxigeninlabeled neurons of gonadotropin-releasing hormone types reveal a novel G protein-coupled receptor (Gpr54) during maturation in cich- lid fish. Endocrinology 145(8): 3613-3618. 
7. Kitahashi T, Ogawa S, Parhar I (2009) Cloning and expression of kiss2 in the zebrafish and medaka. Endocrinology 150(2): 821-831.

8. Li S, Zhang Y, Liu Y, Huang X, Huang W, et al. (2009) Structural and functional multiplicity of the kisspeptin/GPR54 system in goldfish (Carassiusauratus). J Endocrinol 201(3): 407-418.

9. Pasquier J, Lafont AG, Leprince J, Vaudry H, Rousseau K et al. (2011) First evidence for a direct inhibitory effect of kisspeptins on LH expression in the eel, Anguilla anguilla. Gen Comp Endocrinol 173(1): 216-225.

10. Tsutsui K, Bentley GE, Ubuka T, Saigoh E, Yin H, et al. (2007) The general and compara- tive biology of gonadotropin-inhibitory hormone $(\mathrm{GnIH})$. Gen Comp Endocrinol 153(1-3): 365-370.

11. Zhang Y, Li S, Liu Y, Lu D, Chen H, et al. (2010) Structural diversity of the gnih/gnih re- ceptor system in teleost: Its involvement in early development and the negative control of LH release. Peptides 31(6): 1034-1043.
12. Trudeau VL (1997) Neuroendocrine regulation of gonadotrophin II release and gonadal growth in the goldfish, Carassiusauratus. Rev Reprod 2(1): 55-68.

13. Popesku JT, Martyniuk CJ, Mennigen J, Xiong H, Zhang D, et al. (2008) The goldfish (Carassiusauratus) as a model for neuroendocrine signaling. Mol Cell Endocrinol 293(1-2): 43-56.

14. Xu J, Huang W, Zhong C, Luo D, Li S, et al. (2011) Defining global gene expression changes of the hypothalamic-pituitary-gonadal axis in female sGnRH-antisense transgenic common carp (Cyprinuscarpio). PLoS One 6(6): 21057.

15. CHEN Ji, HU Wei, ZHU Zuo Yan (2012) Progress in studies of fish reproductive development regulation. Chin Sci Bull 58(1): 1-16.

16. Zohar Y, Muñoz-Cueto JA, Elizur A, Kah O (2010) Neuroendocrinology of reproduction in teleost fish. Gen Comp Endocrinol 165(3): 438-455.

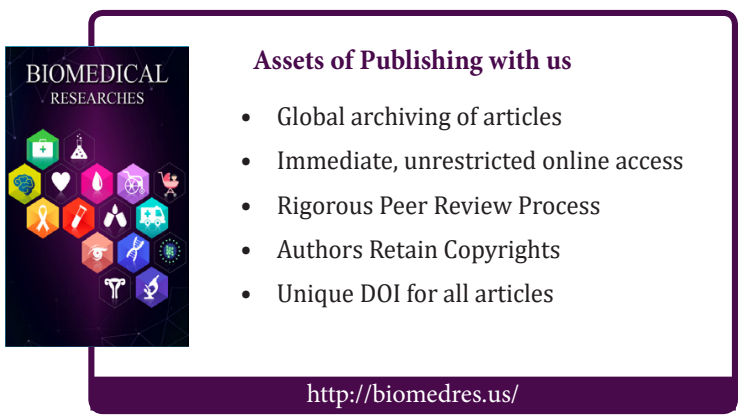

